\title{
Functional treatment of maxillary hypoplasia and mandibular prognathism
}

\author{
Ben Younes-Uzan Carine ${ }^{1 *}$ and Benichou Laurence ${ }^{2}$ \\ ${ }^{1}$ ODF Qualified Specialist, Former pediatric orthodontic consultation assistant at Robert Debre Hospital Paris private practice 60, cours de Vincennes, 75012 \\ Paris, France \\ ${ }^{2}$ ODF Qualified Specialist, Bois-Colombes private practice 4, rue Moulin Massé, 92270 Bois Colombes, France
}

\section{Summary}

Poor development of the maxilla leads to mandibular overdevelopment in the 3 planes of space.

The insufficient growth is fully amenable to correction and increase, since the child is young and still has residual growth, which will result in the stabilization of the results that are achieved.

The use of functional appliances allows the maxillary teeth to receive masticatory stimuli and for the maxillary arch to develop, catching up with its "delay", achieving this simultaneously along with mandibular repositioning.

A functional appliance harnesses the "functions" that are characteristic of living tissue to achieve its effects. Such "functions" are totally ineffective on a typodont.

The function that will be stimulated by the appliance in the mouth is mastication, and the dysfunction to be inhibited is suction.

In this way, case management will be commenced early, and the system will require fewer mechanical appliances in order to be effective.

Nevertheless, functional and mechanical appliances can be used together to achieve better treatment results later [1-3].

The maxilla, due to its adaptive capabilities, in the face of the strong pounding force of the mandible, is the most responsive bone to this type of treatment.

The apparatus will increase the vertical dimension of occlusion to increase the vertical growth of the jaws and will get the teeth moving, which is accompanied by their proprioceptive stimulation from the appliance and that of the tongue.

Complex mandibulomaxillary disharmonies can thus be treated more simply for both the practitioner and for the patient.

\section{Diagnosis and etiology}

We will present 3 clinical cases of children where the maxilla is hypoplastic, and where the non-contained mandible is prognathic.

The first case is a 5 years 8 months old Antillean girl, presenting an articulated complete cross-bite of the whole arch (Figure 1). The maxilla is hypoplastic in the anteroposterior and transverse directions, without any lateral deviation of the mandible, the overbite is reversed but normal in quantity.
This is at primary teeth stage, without any diastemas, the lower teeth are tipped lingually to try to compensate for the discrepancy.

This is genetic on the father's side (Figure 2).

Lateral head-film radiography shows a skeletal class III and on a panoramic film there is a lack of room for the permanent teeth at both arches (Figures 3-9), the 15 and 25 are not visible at this stage and are delayed in their mineralization.

Treatment to make up for late growth will give the adult teeth room for their future positions.

The second case is a 6 years 4 months old boy consulting for a mandibular prognathism (Figure 10).

The mandible extends beyond the maxilla to the front and to the right. As the maxilla enters the mandible to the right before any premature contact generating a deviation, the transverse offset is already visible on the front view, in the rest position.

The smile reveals the lower teeth and is like a grimace.

Occlusion shows endognathy and maxillary hypoplasia with right mandibular prognathism and laterognathism.

The mandibular protrusion is always more obvious on the lateral radiograph which is in occlusion (Figures 11-16) than on the photo, in the rest position, therefore not in occlusion, with the mandible slightly open vertically. On the panoramic radiography, the lack of space is more significant in the upper arch, reflecting its lack of development; the three-dimensional expansion of the arch will give room for the adult teeth.

Case 3 is that of an 11-year-old boy with premaxillary hypoplasia leading to a mandibular prognathism (Figure 17).

Occlusion is reversed on the 4 incisors; the maxillary canines have no room and will erupt in vestibular ectopia. Here again the lack of growth of the maxilla leads to a lack of room for the adult teeth.

The second premolars are not yet in the arch (Figure 18).

${ }^{\star}$ Correspondence to: Ben Younes-Uzan Carine, ODF Qualified Specialist, Former pediatric orthodontic consultation assistant at Robert Debre Hospital Paris private practice 60, cours de Vincennes, 75012 Paris, France, E-mail: drbenyounesuzan@orange.fr

Received: May 02, 2020; Accepted: May 25, 2020; Published: May 27, 2020 

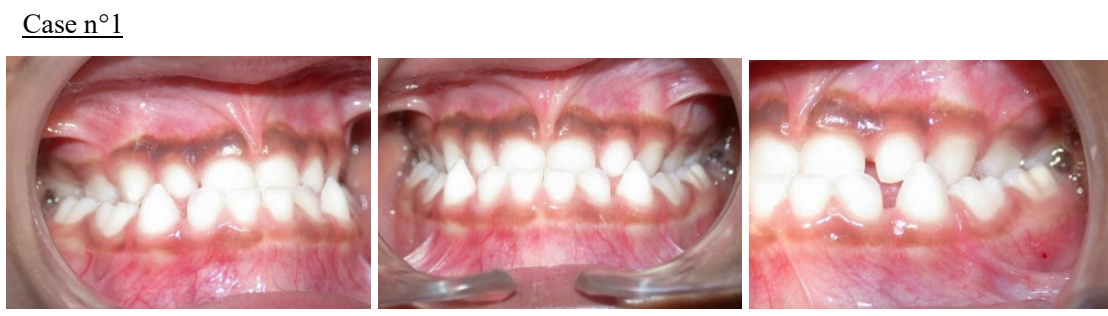

Figure 1. Pretreatment intraoral photographs

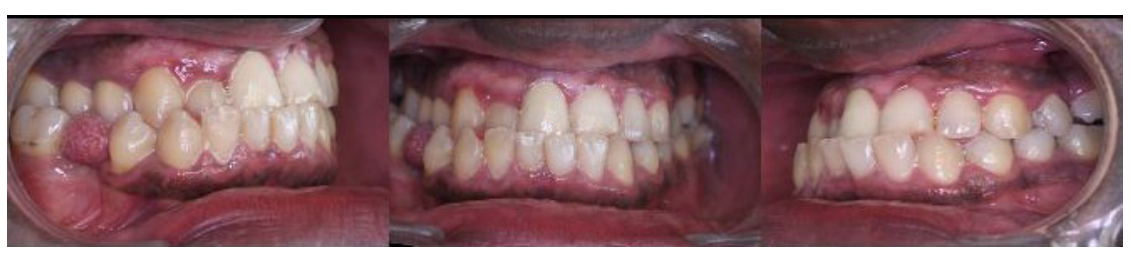

Figure 2. The father's mouth intraoral photographs
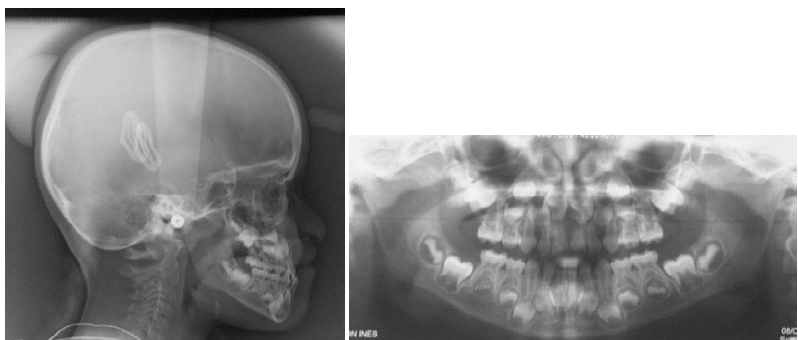

Figure 3. Pretreatment radiographs
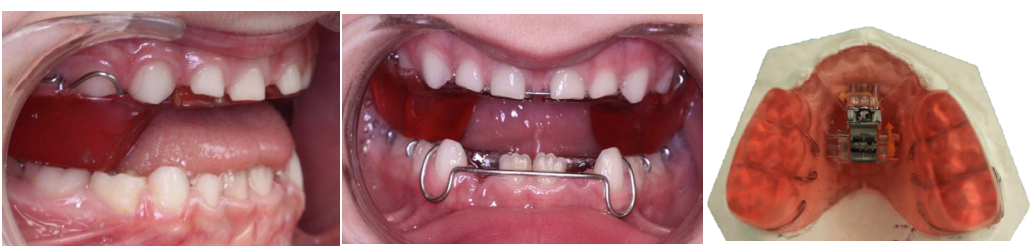

Figure 4. Different functional devices used for similar cases
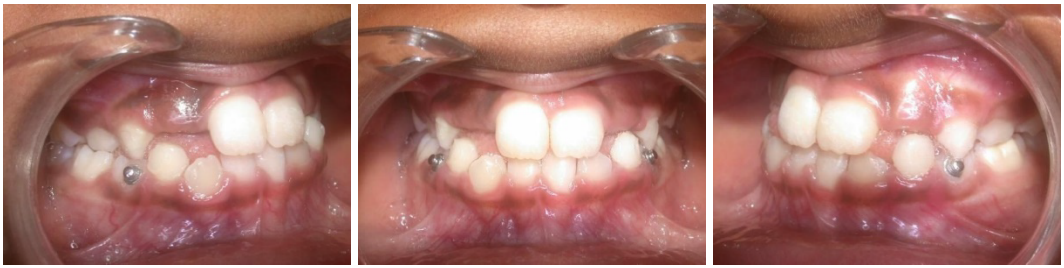

Figure 5. Intraoral photographs at the end of the $1^{\text {st }}$ phase of orthopaedic treatment
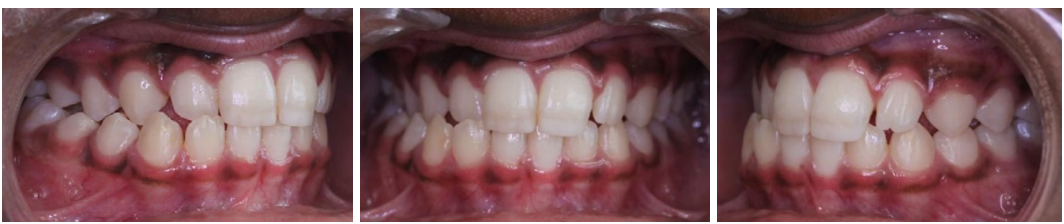

Figure 6. Intraoral photographs before orthodontic treatment 

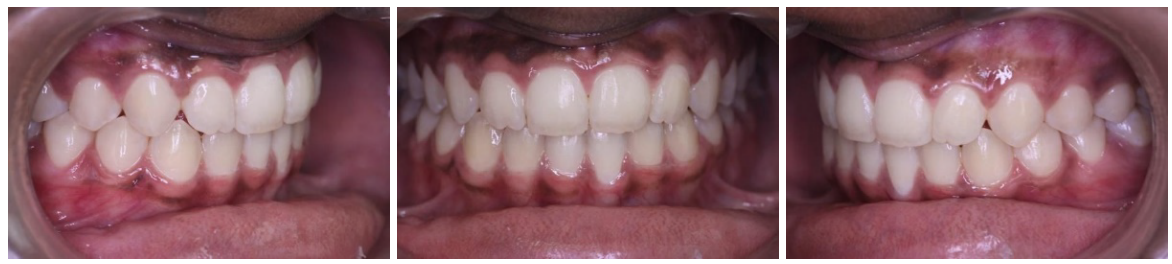

Figure 7. Intraoral photographs after passive self-ligating treatment

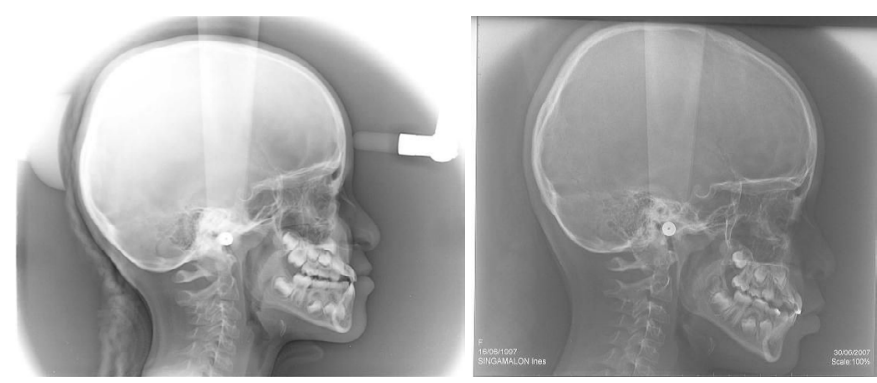

Figure 8. Profile radiographs after occlusion transition and 2 years later

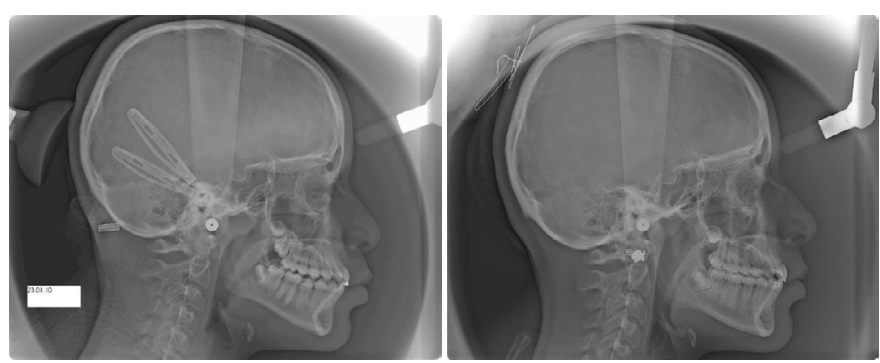

Figure 9. Profile radiographs before and after the brackets treatment

\section{Case ${ }^{\circ} 2$}
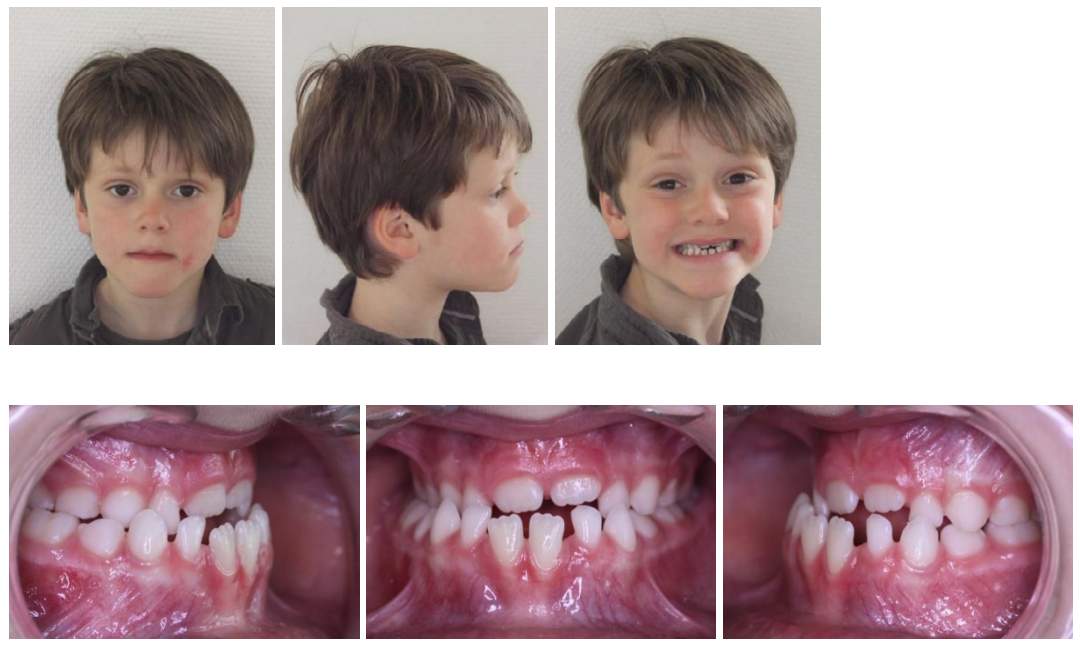

Figure 10. Pretreatment facial and intraoral photographs 


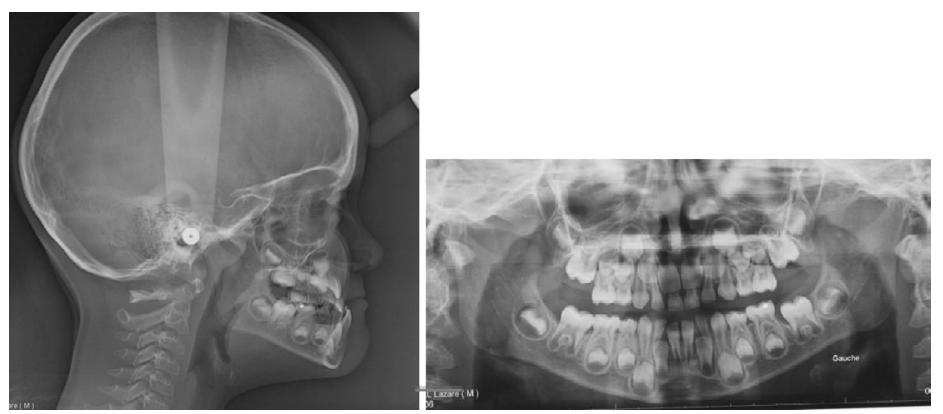

Figure 11. Pretreatment radiographs
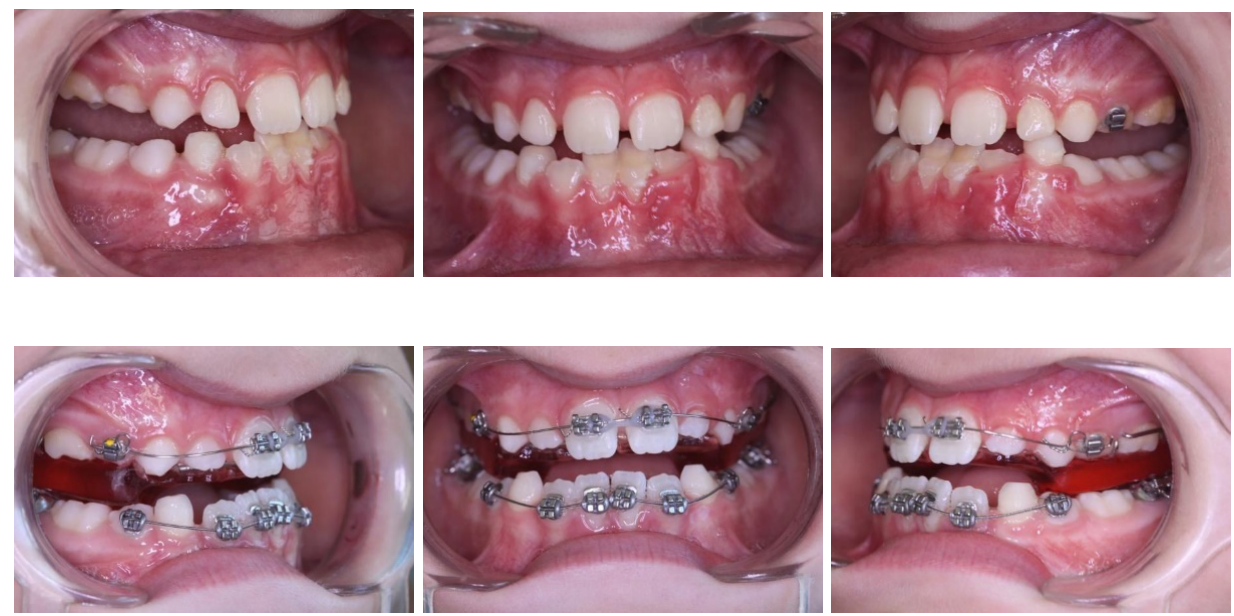

Figure 12. Intraoral photographs upon incisor coverage
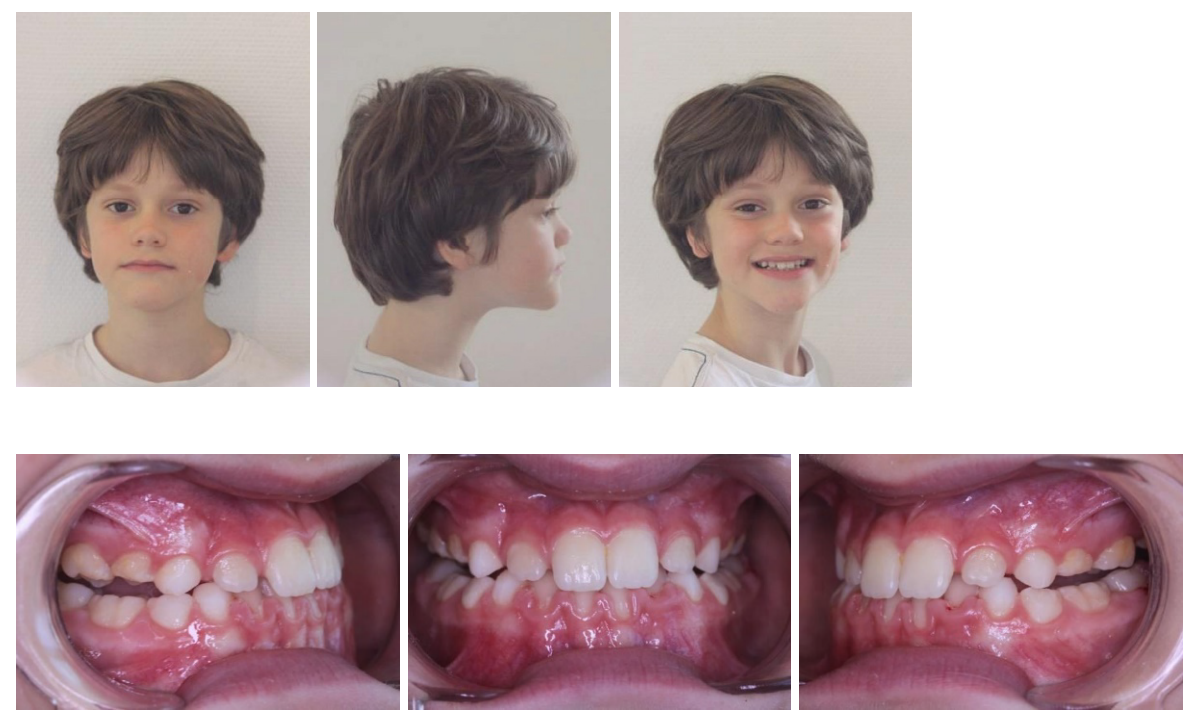

Figure 13. Facial and intraoral photographs at the end of the treatment 

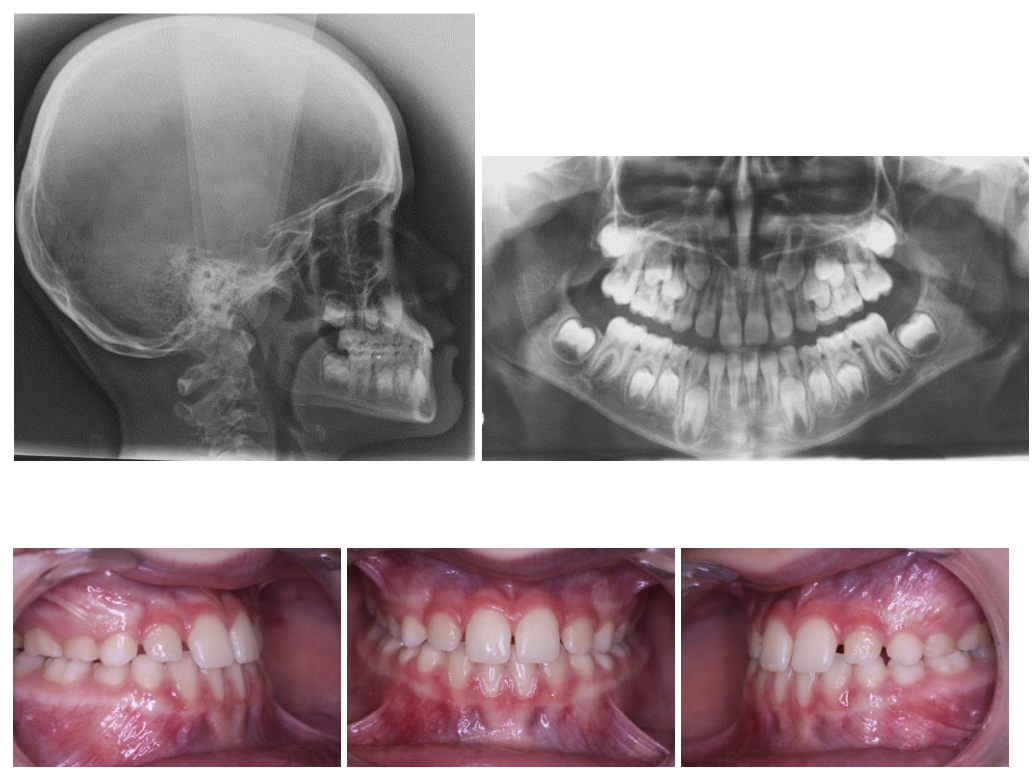

Figure 14. Radiographs and intraoral photographs 3 months later
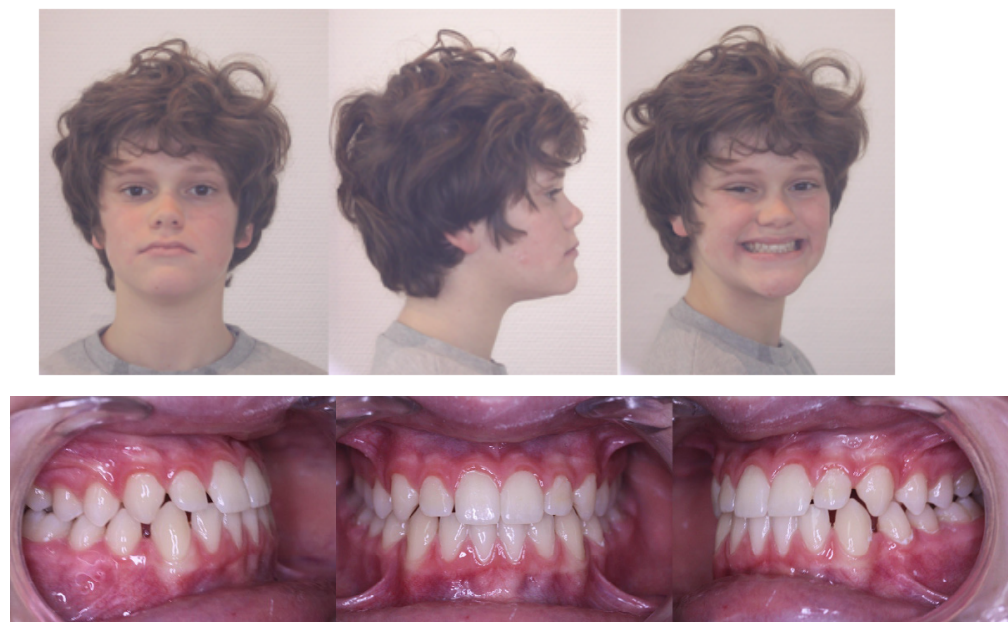

Figure 15. Facial and intraoral photographs 4 years later

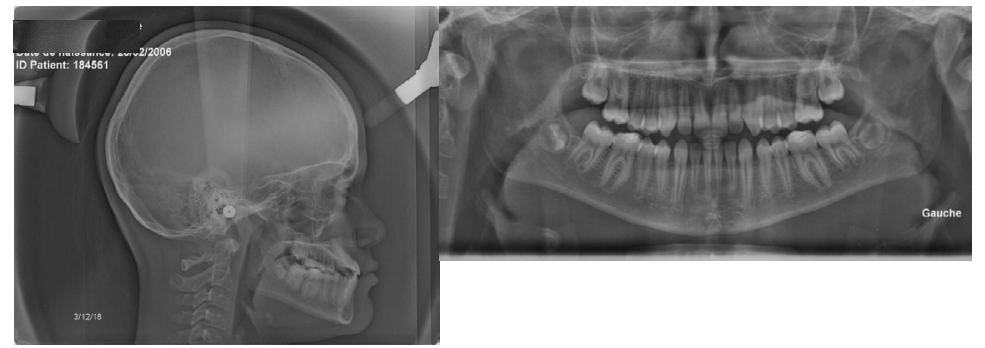

Figure 16. 4 years later radiographs 

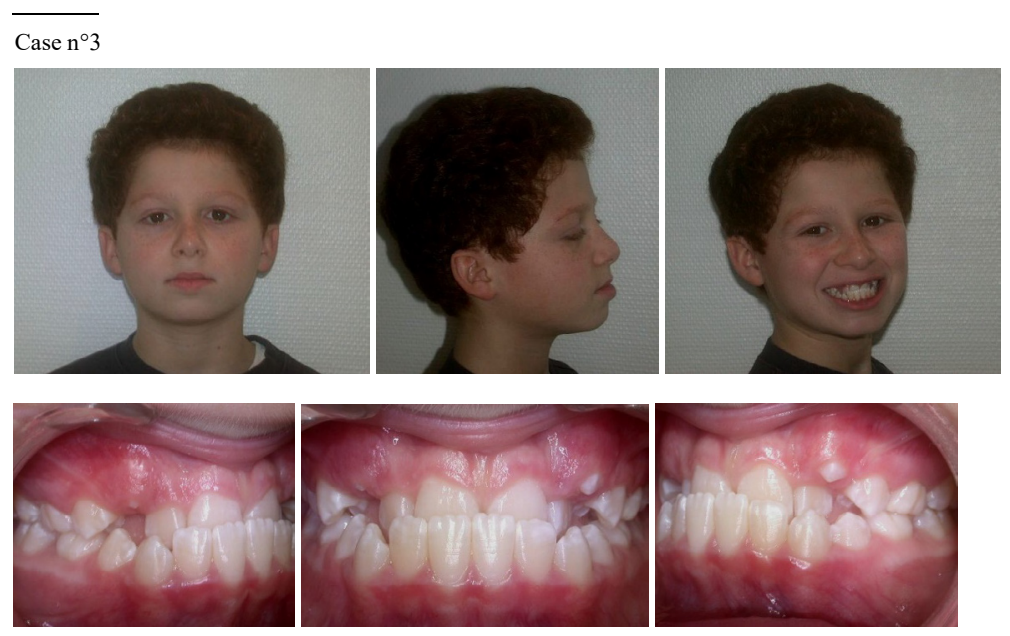

Figure 17. Pretreatment facial and intraoral photographs

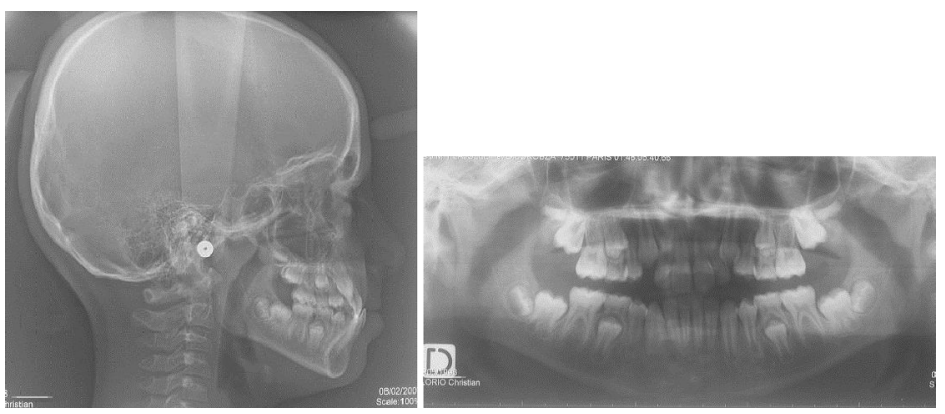

Figure 18. Pretreatment radiographs

In addition to genetic factors, the lack of development of the maxilla associated with mandibular overdevelopment is found in cases of a low tongue. All the factors which lead to the tongue being in the mandibular arch instead of being against the palate are predisposing factors: shortness of the lingual frenum, mouth breathing requiring a free oral channel for the passage of the air, use of the bottle for a long time in infancy and generalized use of the pacifier lasting long after the baby teeth have come through.

The bottle and the pacifier must be stopped before starting the treatment to break the sucking habits [4-10].

\section{Treatment objectives}

In the case of a cross-bite, mandibular growth is uncontrolled and the mandible can overdevelop, anteriorly, downwards, and transversely, generating a mandibular prognathism.

Upper teeth in cross-bite do not receive the proprioceptive stimuli of the opposing arch and without medical management, the maxilla remains in hypoplasia, and the discrepancy cannot spontaneously improve.

These malocclusions are therefore evolving pathologies, which may be diagnosed early from the primary tooth stage where no spontaneous improvement is possible, and therefore a lack of treatment would make it worse and would represent a missed opportunity.

In growing children, no structure has reached its final size. The jaw to be treated is the arch in "delayed" development, which in this case, is the upper arch.
The treatment of the maxilla allows for the control and redirection of the growth of the mandible, thereby preventing the development of a skeletal anomaly with facial deformity.

We want to get a positive buccal relationship in the 3 dimensions (or planes) of space.

The presence of the 1st molar is not a requirement in order to start treatment $[8,10]$. Adult teeth are still preserved despite the shortage of apparent space due to the lack of skeletal development, each dental organ: alveolar-bone-ligament bone, participates in the development of the bone structure: skeletal base + alveolar bone, single-maxillary extractions would worsen the skeletal deficit by an alveolar deficit.

\section{Treatment alternatives}

In general, this type of dysplasia, if not intended for surgery, is treated by an associated facial mask, and for transverse expansion, with maxillary expansion.

\section{Treatment progress}

For the 1st case, we utilized an apparatus with molar bite-blocks and expansion screw(s) in the maxillary arch (Figure 4). The posterior occlusal coverage is the key to treatment, they cover the palatal and occlusal surfaces of the teeth distal to the canines.

The effective height in class III's, in order to obtain a maxillary overbite is for the mandible to be sufficiently lowered, thus retracted to simulate normal inter-incisal ratios in the anteroposterior direction; the more forward is the mandible the higher the height must be. This 
is important so that in occlusion, the lower incisors are sufficiently retruded [5].

The device must be worn permanently, except when cleaning, to make it possible to completely lose the pathological proprioception and to acquire a new one, wearing it during meals is in fact essential. One to two days of adaptation for chewing is needed during which simpler food is recommended.

In addition to the lifting, the device is equipped with a median actuator (screw) acting transversely and either an anterior screw for the 4 incisors, or a retro-incisal omega arch maintaining anterior support against these teeth. With the primary molars enveloped by resin, the displacement of these teeth and their succedaneous dental organs are gradually performed in homeopathic manner, without a parasitic effect, and the mid-palatal suture is also mobilized. This expansion makes room for the adult teeth. Thanks to the resin going between and against the upper teeth, the stimuli of the mandibular masticatory movements will be transmitted to all teeth, including the teeth which were not functional because of the cross-bite.

As there is no more interference, chewing can be alternated between the right and left sides. Anterior brackets make it possible to align and tighten the incisors.

A mandibular plate allowing a slight expansion will move the incisors back to get a positive overjet/overbite.

When the overbite/overjet is normalized, the posterior occlusal overage is gradually thinned and the plate is removed (Figure 5).

In order to preserve the results thus obtained, as a transitional support, the last remaining primary molars are raised, and the 11 and 21 are lengthened.

The patient is re-evaluated when they have their permanent teeth, in order to complete the treatment (Figure 6). It is observed that the anteroposterior direction is better maintained than the transverse direction, and the right side is in underbite from 15 to 12 .
The treatment is finished with passive self-ligating multi- brackets (Figure 7).

Treatment of the 2nd case began with a device with large molar planes for transverse and anterior maxillary expansion. Thanks to the significant lifting of the occlusion, a maxillary overjet is obtained, a class III elastic is placed on the left to help the realignment.

When the upper teeth cover the lower ones (Figure 12), the height of the bite blocks are progressively reduced so as not to leave a lateral open bite. The maxillary central incisors are brought together to give space for the lateral incisors, and the mandibular brackets improve the overbite/overjet and the position of the lower incisors. In the photo the posterior bite blocks have already been thinned (Figure 12) by more than half their effective starting height. The rings and the device are removed 3 months later (Figure 13), in June, for the summer holidays, then the patient was seen at the beginning of September (Figure 14).

The third case is equipped with a plate with molar occlusal coverage and anterior and transverse expansion screws (Figure 17). A lingual arch makes it possible to preserve the E-space while maintaining 36 46 , and to allow the anteroinferior teeth to spontaneously move back. Photos (Figure 18) were taken during the same session, when the incisors are covered, with and without a device. The molar occlusal coverage is reduced to reach a height corresponding to at least the molar open bite so as not to have any ejection at closing. Bringing the incisors together then frees up room for the canines (Figure 19). The device is progressively removed as well as the lingual arch, and the alignment is completed with brackets bonded to all the teeth (Figure 20).

\section{Discussion}

As is often case in older people who are no longer growing the loss of the vertical dimension of occlusion due to the bilateral loss of posterior teeth leads to a class III inter-maxillary relationship. In a growing child, the increase in the vertical dimension of occlusion by occlusal coverage on the posterior teeth will facilitate the impediment of mandibular protraction and promote maxillary growth as is the principle used here $[1,5,6]$.
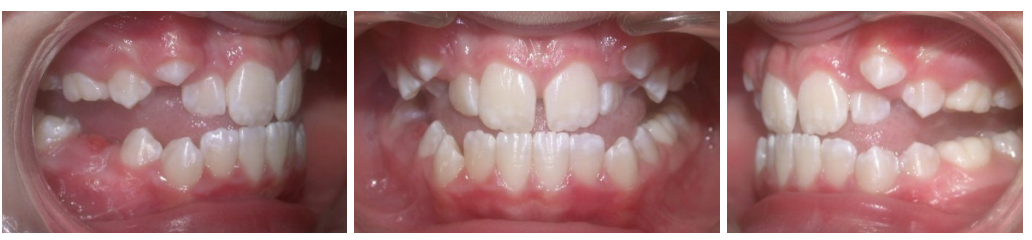

Figure 19. Intraoral photographs after 2 months
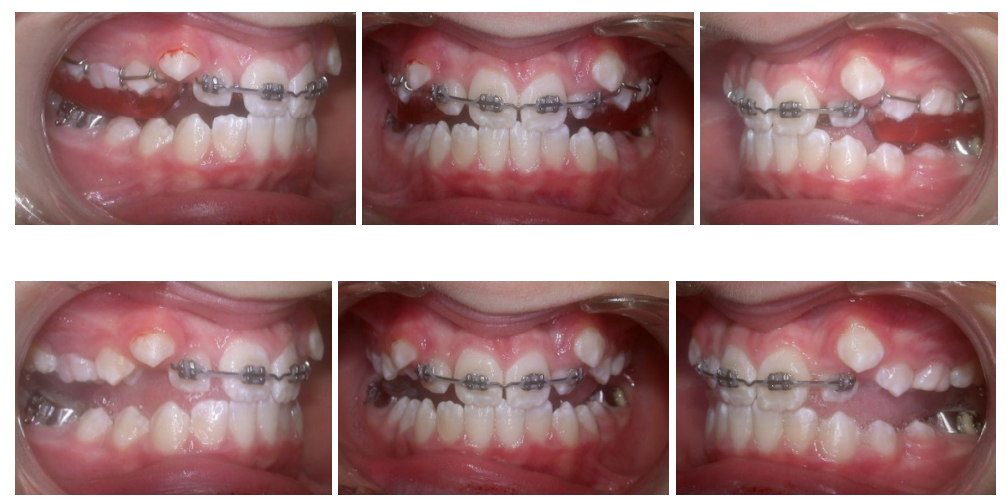

Figure 20. Intraoral photographs when incisor coverage is achieved with and without removable device 
The increase in occlusal height will be achieved through the use of a removable plate with molar occlusal coverage that can be used in children from the time of the primary teeth $[10,8]$.

With the appearance of the adult teeth, all other conventional mechanical orthodontic systems can be utilized. The interposition of flat resin planes between the molars on a plate in contact with all the maxillary teeth allows all the upper teeth to receive the stimuli of mastication.

The response to these stimuli will be according to Planas's laws of development [11], whereby the mobilization of a tooth from a maxillary or mandibular embryologic bud leads to a growth response from all the teeth in that bud; thus the teeth of the 3 maxillary embryonic buds and the 2 mandibular embryological buds can respond.

The action of the device is expressed in the 3 dimensions of space, the regular activation takes place thanks to the actuators.

The significant height of the bite-planes modifies the direction of masticatory forces [5]; vertical forces become oblique, with an anteroposterior component that pushes the maxilla forwards, as if it was being pulled by a face mask.

With the resin being in contact with the lingual surfaces of the upper incisors, the functional mobilization of the occlusal strike (Delaire) [8] and the friction of mastication (Planas) [11] are transmitted to these teeth, providing the energy required to produce the expansion of this arch.

The earlier the expansion in the 3 dimensions of space, the less there are facial consequences, Delaire explained the role of the forces of occlusion, vital at the time of the arrival of the teeth that "empty" the maxilla and participate in sinus development; providing an external exo-face structure and an endo-face internal structure.

While chewing pushes the exo-face outwards, the internal sutures pull the endo-face inward, this dynamic actively participates in the pneumatization of the face and the construction of its volume.

The direction of the ligaments and muscles, particularly the lateral pterygoid muscles, is modified by the anterior movement and descent of the condyle within the glenoid cavity (fossa). Their axis is transformed, initially horizontal, and then oblique. As the axis reaches a vertical position, the muscles lose a portion of their propulsive activity on the mandible.

The tongue, which is generally very powerful in these patients, can carry out its centrifugal action on the superior incisors, which are no longer restricted by the occlusion. The lateral wedges of the resin mould will increase the volume available to it within the dental arcades, which will be subject to their thrust.

Since their thickness is greater than the inter-occlusal distance, lateral lingual interposition between the arches is prevented and dental proprioception is stimulated, because the posterior teeth are in contact again through molar bite-planes.

The inferior orbicularis muscle will no longer exercise constraints on the freed superior incisors; rather, it will do so on the mandibular teeth.

The superior orbicularis muscle is less tonic and offers less resistance to the lingual thrust against the maxillary incisors.

The appliance also has a role in preventing dysfunction, with real antagonism existing between the tongue and the teeth [4].
In the newborn, the tongue spreads over the alveolar ridge for the baby to feed and swallow in suction-deglutition. With time, as the teeth erupt and rub on each other for mastication, the tongue retracts "so as not to get bitten", except when the infant persists in sucking habits or habitual mouth-breathing, keeping the tongue in a low position, while the teeth remain at a distance. Everything that goes into the mouth gets bitten except the tongue (in the absence of anesthesia), which is covered with proprioceptors and nociceptors.

In the context of this antagonism, two therapeutic approaches exist: encouraging the tongue to retract by using re-training techniques and lingual re-positioning [7], or making the teeth function in such a way that lateral rubbing movements and masticatory stimuli are transmitted to all of the teeth, including those in malocclusion.

When the tongue no longer lies between the arches, it can be positioned on the palate, which (favors) nasal respiration as well as maxillary expansion, since the nose is the roof of the mouth and the expansion of the palate necessarily creates better nasal airflow [6]. A perfectly adapted palatal resin mould also facilitates the restoration of proprioception to the dorsal surface of the tongue, which can move to a higher position without receiving pathological support. The bottom resin surface will be thinned over time by expansion in order to give the palate a shape that is adapted to the lingual dome.

The device allows transverse action on the lateral cross-bite also, because the resin interposed between the teeth will release the upper teeth of the pathological occlusion which was a hindrance to the arch's development and mastication becomes possible alternating on both sides.

The molar elevation can also contribute to closing the vertical direction [2] by entering the posterior areas covered with resin and enabling the anterior teeth to spontaneously become extruded.

\section{Treatment results}

In the first case, looking at the profile radiographs, we note that the upper central incisors were in the pro-alveolar stage at the end of the first phase of treatment (Figure 8), since normalized occlusion was obtained early on (when the incisors arrived), growth will spontaneously improve their inclination by shifting the roots (Figure 9).

Earlier functional occlusion allows growth to continue to improve outcomes.

For the second case, we note that thanks to the treatment, the face has found harmony and symmetry.

After treatment the patient no longer smiles displaying the lower teeth, but with displaying the upper incisors (Figure 13).

The lifting device allowed the maxilla to be placed over the mandible, and previously bonded brackets aligned the teeth. The lack of space for the adult teeth spontaneously changed (Figure 14).

The patient is re-evaluated with their adult teeth at 12 years 9 months (Figure 15) with a radiological assessment (Figure 16), to undergo a multi-banded finish with the early orthopedic results having been maintained.

The third case, undertaken later on, shows that the functional device allowed the anterior zone of the maxilla to start growing again. The brackets are placed as soon as the positive overbite/jet is obtained and the same time that the height of the planes is reduced (Figure 18). 
The patient is seen at 22 years old 8 months (Figures 21-23), at the request of the practitioner, accompanied with a radiological assessment (Figure 24).

The wisdom teeth were extracted, the results are generally maintained with the exception of the articulated 23/33, no retention wire was bonded at the time to prevent periodontal problems on the incisors in case of possible recurrences (Figures 25 and 26).

It is noted, however, that there is a lack of volume in the middle part of the face and maxilla, compared to earlier cases, in which, in adulthood, no evidence of the initial malocclusion is detectable on the face.
The use of a lingual arch to block the 6 (molars) in the treatment of the class III is perhaps responsible for the secondary mesialization of these teeth and the lack of space which appeared afterwards for the lower canines.

\section{Conclusions}

Functional devices can promote the growth of the maxilla in the 3 dimensions of space by modifying the vertical dimension of occlusion and by giving the mandible freedom of movement. Their use can be as early as the milk teeth stage, but they can be connected to all the devices of classical orthodontics to potentiate their effects.
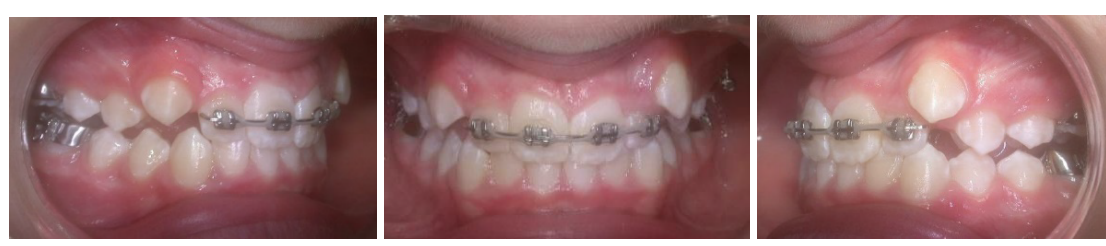

Figure 21. Intraoral photographs when the removable device is removed
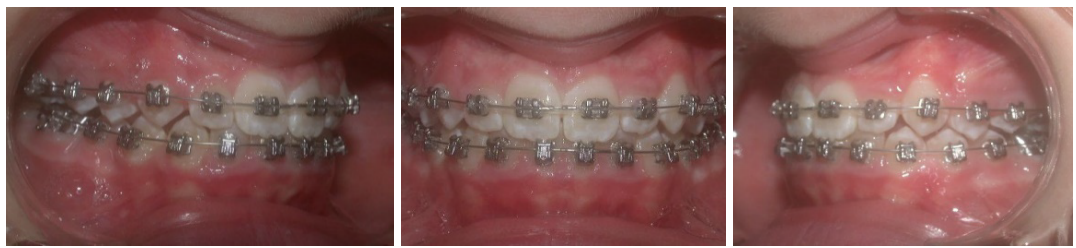

Figure 22. Intraoral photographs with braces
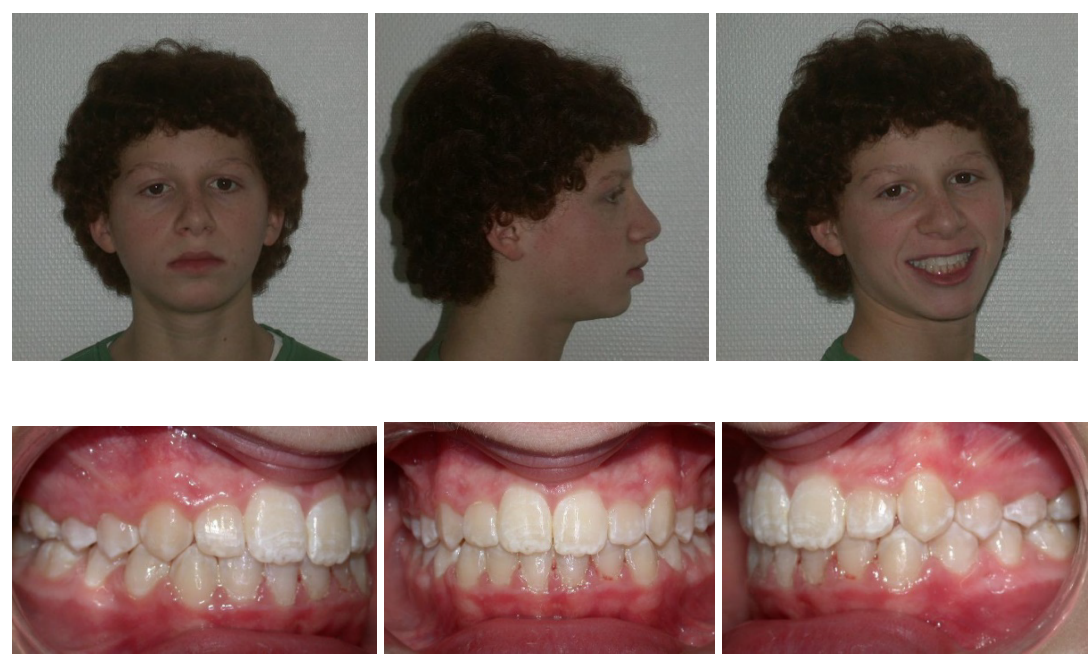

Figure 23. Facial and intraoral photographs after treatment
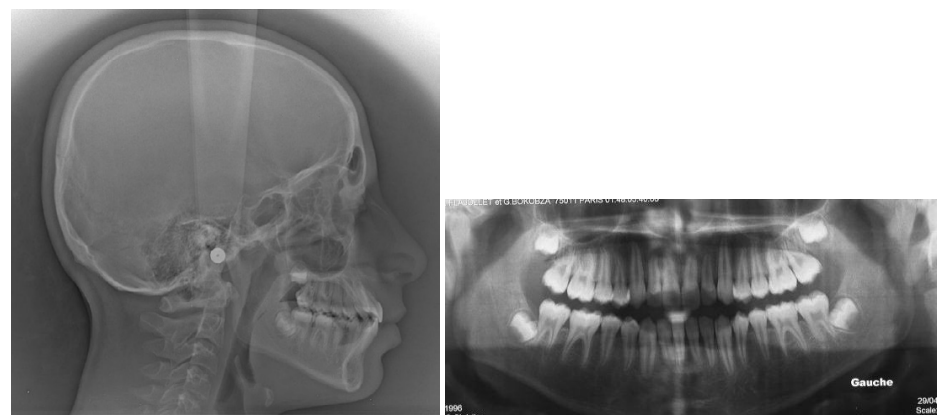

Figure 24. Radiographs after laying the brackets 

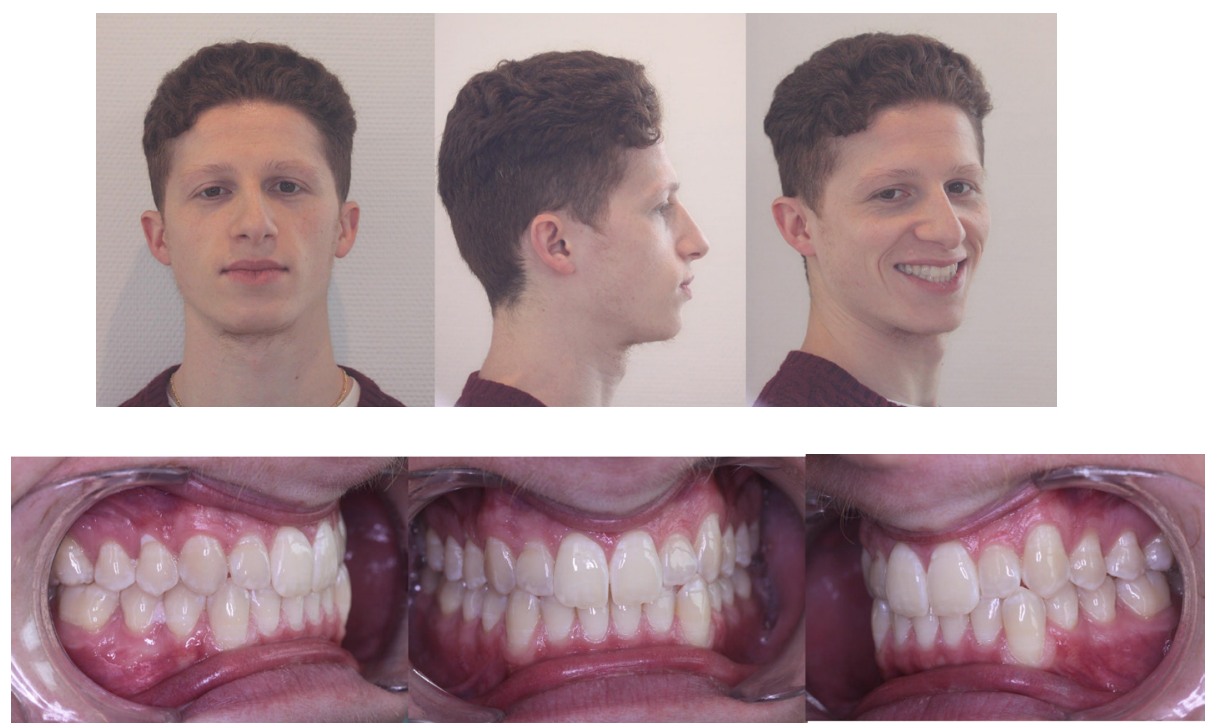

Figure 25. Facial and intraoral photographs 10 years after treatment, 9 years post retention
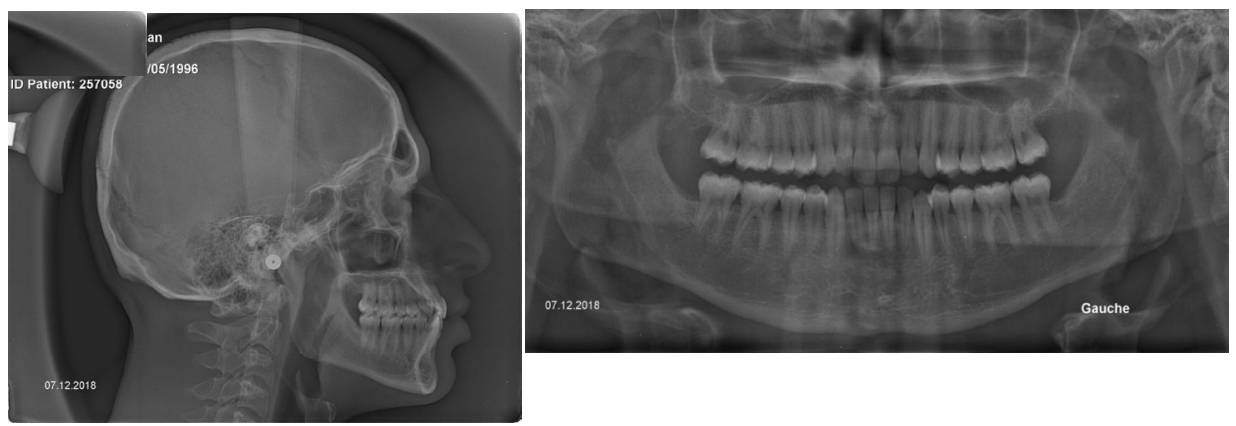

Figure 26. Radiographs 10 years after treatment, 9 years post retention

The increased growth of the maxillary gives place to the adult teeth, allows a positive redirection of the mandibular growth resulting in the simple resolution of very complex cases.

The results are far better when the subject is young, before the lateral maxillary teeth arrive.

\section{Highlights}

This is an article showing 3 cases to illustrate the author's use of a removable resin appliance to increase a patient's posterior vertical dimension. This done in order to eliminate interferences and to utilize the forces of mastication to induce increased anteroposterior proprioceptive effects on the maxilla allowing for the maxilla of a growing patient to achieve correction in Class III cases.

Comprehensive before, during and after treatment records are shown. The authors have many additional cases of patients treated from the ages of 4 to 16.

\section{References}

1. Ben Younes-Uzan C (2007) Proposition non conventionnelle de traitement des classes III. L'orthodontie française 82( $\left.\mathrm{n}^{\circ} 2\right)$ : 133.
2. Ben Younes- Uzan C. Orthopaedic treatment of class III by stimulation of the growth of the jaw http://aos.edp-dentaire.fr/articles/aos/abs/2018/01/aos2018287article6/ aos2018287article6.ht ml)

3. French version : source

4. http://aos.edp-dentaire.fr/articles/aos/abs/2018/01/aos2018287article6/ aos2018287article6.ht ml

5. Fellus P (2003) Orthodontie précoce en denture temporaire. Paris : CDP.

6. Deshayes MJ (2006) L'art de traiter avant 6 ans. Préface Baudinet V. Paris : Cranexplo.

7. Deffez JP (1971) Prognathies mandibulaires. Propositions thérapeutiques. Paris : Julien Prélat.

8. Deffez JP (1979) Bases de l'étage moyen de la face. Les sites et les facteurs de croissance du maxillaire: données actuelles. Act Odonto-Stomatol 128: 647-668.

9. Planas P (1992) La Réhabilitation neuro-occlusale. Paris: Masson.

10. Delaire J (1997) Maxillary development revisited: relevance to the orthopaedic treatment of Class III malocclusions. European Journal of Orthodontics 19: 289-311.

11. Couly G (1989) La langue, appareil naturel d'orthopédie dento-faciale pour le meilleur et pour le pire. Rev. Orthop. Dento-Faciale 23: 9-17.

12. Deffez JP, Fellus P, Gérard C (1998) Rééducation de la déglutition salivaire. Paris: CdP.

13. Ben Younes-Uzan C (2017) Fermeture des infraclusions par mastication sur les dents postérieures. Rev Orthop Dento-Faciale 51: 447-455.

Copyright: $\odot 2020$ Carine BY. This is an open-access article distributed under the terms of the Creative Commons Attribution License, which permits unrestricted use, distribution, and reproduction in any medium, provided the original author and source are credited. 